\title{
Efficacy and Patient Satisfaction of Dipeptidyl Peptidase-4 Inhibitor After Switching From Once-Daily DPP-4 Inhibitor to Once-Weekly Regimen
}

\author{
Katsunori Suzukia, b, Kazuki Hasegawa ${ }^{\mathrm{a}}$, Mio Watanabe ${ }^{\mathrm{a}}$
}

\begin{abstract}
Background: We administered once-weekly dipeptidyl peptidase-4 (DPP-4) inhibitor (W) (used omarigliptin as W in this study) to patients with type 2 diabetes taking once-daily DPP-4 inhibitor (D), and investigated efficacy, safety and patient satisfaction before and after switching to W.
\end{abstract}

Methods: W was administered to 182 patients with type 2 diabetes taking D (used sitagliptin as D in this study), who had been visiting our hospital on an outpatient basis; 164 (90.6\%) of these patients requested to switch medications. Of these 164 patients, this study investigated 153 who requested to continue taking W. Hemoglobin A1c (HbA1c) levels, body weight, blood pressure and a questionnaire survey (Diabetes Treatment Satisfaction Questionnaire (DTSQ)) were evaluated in these patients.

Results: Patient characteristics were as follows: age, $63.9 \pm 10.3$; male/female ratio, 93:60; duration of diabetes, $14.9 \pm 7.7$ years; and body mass index (BMI), $25.5 \pm 4.2 \mathrm{~kg} / \mathrm{m}^{2}$. After switching to $\mathrm{W}, \mathrm{HbA} 1 \mathrm{c}$ levels changed from $7.41 \pm 0.7 \%$ to $7.36 \pm 0.9 \%$, which was not statistically significant. Changes in body weight, BMI, and systolic and diastolic blood pressure were also not significant. On the DTSQ, satisfaction of Q1 significantly increased $(\mathrm{P}<0.01)$. The score for lifestyle assessment did not significantly change, but compliance significantly improved $(\mathrm{P}<0.001)$.

Conclusion: This study revealed that $90 \%$ of patients taking D elected to switch to W. Moreover, patient satisfaction and compliance improved after switching to W. Increased satisfaction appeared to be influenced by improved blood glucose control, but was not associated with compliance. Switching from $\mathrm{D}$ to $\mathrm{W}$ did not affect HbA1c levels but improved patient adherence.

Keywords: Adherence; Satisfaction; Compliance; Weekly DPP-4 in-

Manuscript submitted May 14, 2018, accepted May 29, 2018

aDivision of Endocrinology and Metabolism, Saiseikai Niigata Daini Hospital, Niigata, Japan

${ }^{\mathrm{b} C}$ Corresponding Author: Katsunori Suzuki, Division of Endocrinology and Metabolism, Saiseikai Niigata Daini Hospital, 280-7 Teraji, Nishi-ku, Niigata, Niigata 950-1104, Japan. Email: katsu-s@ngt.saiseikai.or.jp

doi: https://doi.org/10.14740/jocmr3456w hibitor; Omarigliptin; Type 2 diabetes

\section{Introduction}

Remarkable progress has been made in medications for treating diabetes in parallel with a global increase in the number of diabetic patients. In the realm of diabetes medications, therapeutic drugs with novel mechanisms of action are continually being developed, although existing drugs with prolonged actions are also rapidly appearing on the market. The addition of such therapeutic drugs to the repertoire of treatment options is anticipated to be beneficial for improving patient adherence.

The first dipeptidyl peptidase-4 (DPP-4) inhibitor was approved in Japan in 2009. DPP-4 inhibitors suppress postprandial hyperglycemia, are less likely to induce weight gain or hypoglycemia, and involve relatively few comorbidity-related restrictions. Therefore, they are frequently used in Japan [1]. In 2015, once-weekly DPP-4 inhibitors (W), trelagliptin and omarigliptin, were approved in Japan before global approval. In clinical trials during drug development, trelagliptin was compared to alogliptin and omarigliptin was compared to sitagliptin. Non-inferiority in efficacy and safety was demonstrated for both drugs compared to the existing daily medications $[2,3]$. It was also recently reported in a meta-analysis that the efficacy and safety of once-weekly medication are comparable to that of existing daily medications [4]. Because onceweekly DPP-4 inhibitors are only commercially available in Japan, their efficacy and safety in general practice have not been established.

The reduced number of doses and tablets in once-weekly DPP-4 inhibitor (W) therapy may ameliorate patient treatment satisfaction and medication adherence, resulting in better longterm blood glucose control. However, no clear superiority in drug efficacy has been demonstrated compared to the existing and familiar once-daily DPP-4 inhibitor (D), and patient interest and assessment of W have been unclear. In this study, we proposed $\mathrm{W}$ to patients with type 2 diabetes taking $\mathrm{D}$ (used sitagliptin as D in this study), and investigated the changes in serum levels of hemoglobin A1c (HbAlc) before and after switching to W (used omarigliptin as W in this study). We also conducted a questionnaire survey (Diabetes Treatment 
Table 1. The Checklist for Life-Style Assessment

\section{Please tell us about your current eating habits and exercise habits.}

1. Do you skip your ordinary meal (for example, skip breakfast, etc.)?

Never: (1 point)

Sometimes or often: (0 points)

2. Do you eat your dinner later than 9 PM?

Never: (1 point)

Sometimes or often: (0 points)

3. Are you taking care of the amount and content of your meal (for example, cutting down sugar, salt or oil, etc.)?

Often: (2 points)

Sometimes: (1 point)

Never: (0 points)

4. Do you have a snack between meals?

Never: (1 point)

Sometimes or often: (0 points)

5 . Do you eat out in the evening?

Never: (1 point)

Sometimes or often: (0 points)

6. Do you drink alcohol?

Never: (1 point)

Sometimes or often: (0 points)

7. How often do you exercise a week?

Every day: (2 points)

1 to 6 days: (1 point)

Never: (0 points)

8. How long do you exercise a day?

More than 1 hour: (2 points)

Within 1 hour: (1 point)

Never: (0 points) (who answered 'never' in question 7)

The sum of the points represented the lifestyle assessment score.

Satisfaction Questionnaire (DTSQ)) to assess satisfaction and compliance.

\section{Materials and Methods}

Patients were enrolled between June and August 2017. W was proposed to 182 patients with type 2 diabetes taking $\mathrm{D}$, who had been visiting our hospital on an outpatient basis and had no changes in their diabetes treatment (e.g., diet, exercise, medication) within the past 12 weeks. Clinical examination was performed as usual at the visit. After being notified of their blood test results (e.g., HbAlc levels) of that day, all patients were given the following information about the once-weekly medication: "There is a drug that has essentially the same therapeutic effects as those of the DPP-4 inhibitor (D) you are currently taking. This drug only needs to be taken once a week. There is very little difference in price. We can switch or con- tinue the current medication. If the new medication does not seem suitable for you, we will switch back to the original drug (D). What would you like to do?"

We evaluated $\mathrm{HbA} 1 \mathrm{c}$ levels, body weight, body mass index (BMI), systolic blood pressure, diastolic blood pressure and patient survey responses before and 8 weeks after switching the patients to W. Three evaluation methods were used in the patient surveys. 1) DTSQ translated by Ishii et al [5] was used to evaluate patient satisfaction. The questionnaire consists of eight questions quantified on a seven-point scale of $0-6$ and is widely used to evaluate treatment satisfaction in diabetic patients. 2) The evaluations of lifestyle were made according to the following checklist (Table 1), which was modified for this study [6].

The sum of the points represented the lifestyle assessment score. 3) Compliance was evaluated on a visual analog scale (VAS) as shown in Figure 1.

This study was conducted in accordance with the Ethi- 


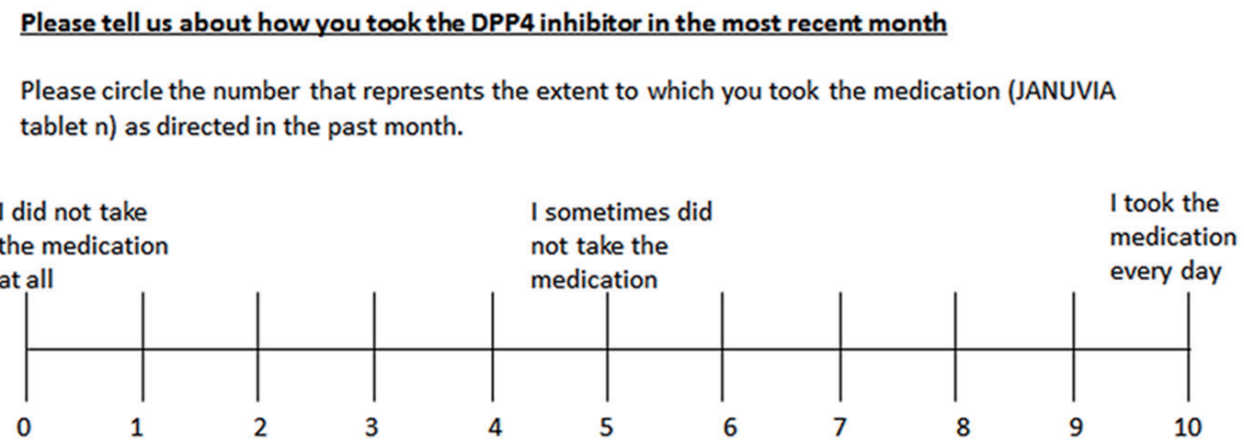

Figure 1. Visual analog scale of compliance with medication schedule.

cal Guideline for Clinical Research of the Ministry of Health, Labour and Welfare after obtaining written informed consent from the patients. We obtained approval from the Institutional Review Board of Saiseikai Niigata Daini Hospital (Approval No. E16-38).

All tests were performed using Microsoft Excel 2016 for Mac, and all data are expressed as the mean \pm standard deviations. Student's $t$-test was used for the comparisons of values between before and 8 weeks after switching to W. P values less than 0.05 were considered statistically significant.

\section{Results}

Patient characteristics are shown in Figure 2. A total of 164
$(90.6 \%)$ patients requested to switch medications from $\mathrm{D}$ to $\mathrm{W}$, after being provided information about W. Of these 164 patients, this study investigated 153 who requested to continue taking W (11 patients requested to switch back to D during the study). Patient characteristics were as follows: age, $63.9 \pm$ 10.3; man/woman ratio, 93:60; duration of diabetes, $14.9 \pm 7.7$ years; BMI, $25.5 \pm 4.2 \mathrm{~kg} / \mathrm{m}^{2}$; estimated glomerular filtration rate, $70.2 \pm 22.6 \mathrm{~mL} / \mathrm{min} / 1.73 \mathrm{~m}^{2}$; HbA1c, $7.4 \pm 0.7 \%$; systolic blood pressure, $122.0 \pm 11.3 \mathrm{mmHg}$; diastolic blood pressure, $72.5 \pm 7.7 \mathrm{~mm} \mathrm{Hg}$; and number of different medications, 5.1 \pm 2.2 (all insulin formulations were considered to be one type) (Table 2).

After switching to $\mathrm{W}, \mathrm{HbA} 1 \mathrm{c}$ levels changed from $7.41 \pm$ $0.7 \%$ to $7.36 \pm 0.9 \%$, which was not statistically significant. Changes in body weight, BMI, and systolic and diastolic blood

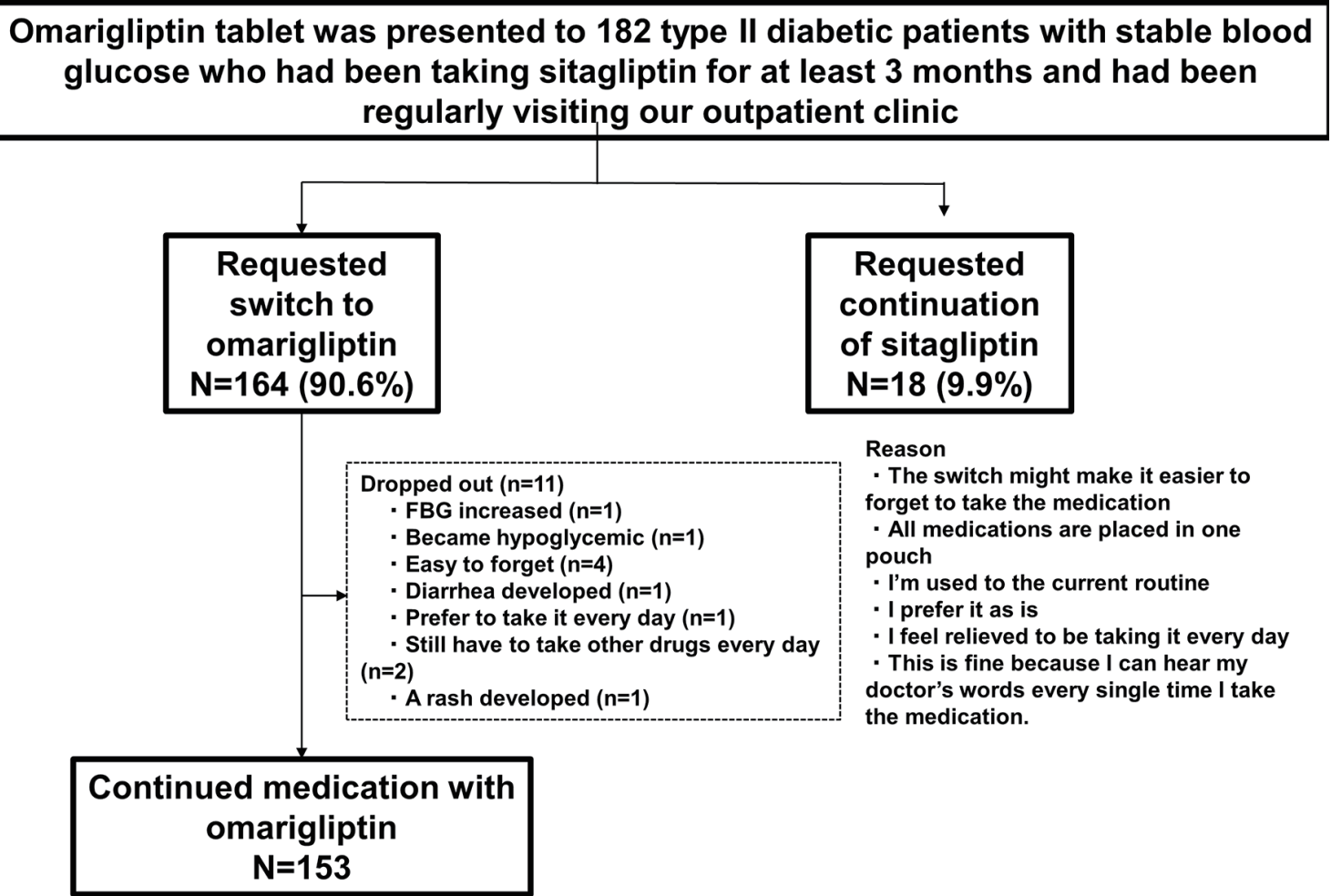

Figure 2. Patient attrition. 
Table 2. Patient Characteristics

\begin{tabular}{ll}
\hline Factors & Mean \pm SD (range) \\
\hline Number of patients $(\mathrm{n})$ & 153 \\
Age (years old) & $63.9 \pm 10.3(28-87)$ \\
Sex (male/female) & $94: 59$ \\
\hline Duration of diabetes (years) & $14.9 \pm 7.7(1-43)$ \\
BMI $\left(\mathrm{kg} / \mathrm{m}^{2}\right)$ & $25.5 \pm 4.2(16.1-39.7)$ \\
Body weight $(\mathrm{kg})$ & $65.2 \pm 9.5(49.2-84.3)$ \\
HbA1c $(\%)$ & $7.41 \pm 0.75(5.7-9.6)$ \\
SBP $(\mathrm{mm} \mathrm{Hg})$ & $122.1 \pm 11.3(94-143)$ \\
DBP $(\mathrm{mm} \mathrm{Hg})$ & $72.5 \pm 7.8(59-88)$ \\
eGFR $\left(\mathrm{mL} / \mathrm{min} / 1.73 \mathrm{~m}^{2}\right)$ & $70.23 \pm 22.9(40.9-121.2)$ \\
Number of drugs $(\mathrm{n})$ & $5.1 \pm 2.2(1-12)$ \\
U-AER $(\mathrm{mg} / \mathrm{gCr})$ & $94.4 \pm 187.9(2.3-873.2)$ \\
\hline
\end{tabular}

Data are presented as mean \pm SD. AER: albumin excretion rate; $B M I$ : body mass index; SBP: systolic blood pressure; DBP: diastolic blood pressure; eGFR: estimated glomerular filtration rate. The insulin preparation counted it for one kind in all.

pressure were also not significant (Table 3).

The questionnaire response rate was $100 \%$ because all participants were patients who had agreed to complete the questionnaire survey. For DTSQ, the satisfaction of Q1 significantly increased $(\mathrm{P}<0.01$; Fig. 3). Recommendation (Q7) tended to increase, but it was not significant. Significant changes in responses were not observed for any of the other questions (Qs).
Table 3. Changes in Clinical Parameters After Switching From Sitagliptin to Omarigliptin

\begin{tabular}{llll}
\hline & Baseline & $\mathbf{8}$ weeks & P value \\
\hline BMI $\left(\mathrm{kg} / \mathrm{m}^{2}\right)$ & $25.5 \pm 4.2$ & $25.3 \pm 3.6$ & $\mathrm{NS}$ \\
Body weight $(\mathrm{kg})$ & $65.2 \pm 9.5$ & $65.7 \pm 9.6$ & $\mathrm{NS}$ \\
HbA1c $(\%)$ & $7.41 \pm 0.75$ & $7.36 \pm 0.79$ & $\mathrm{NS}$ \\
SBP $(\mathrm{mm} \mathrm{Hg})$ & $122.1 \pm 11.3$ & $127.6 \pm 11.9$ & $\mathrm{NS}$ \\
DBP $(\mathrm{mm} \mathrm{Hg})$ & $72.5 \pm 7.8$ & $75.5 \pm 9.7$ & $\mathrm{NS}$ \\
\hline
\end{tabular}

Data are presented as mean \pm SD. BMI: body mass index; SBP: systolic blood pressure; DBP: diastolic blood pressure; NS: not significant by Student's paired $t$-test.

The association between the number of different medications before switching and the extent of change in satisfaction (Q1) was analyzed, but a correlation was not found (data not shown). Subsequently, patients were divided into two groups: those with increased satisfaction and those with decreased satisfaction on Q1, and the clinical parameters were compared between the two groups (Table 4). In comparing the increased satisfaction $(n=46)$ and decreased satisfaction groups $(n=24)$, the former group had significantly improved $\mathrm{HbA} 1 \mathrm{c}$ levels compared to the latter group $(-0.154 \pm 0.32 \%, 0.146 \pm 0.49 \% ; \mathrm{P}<0.01)$. The score for lifestyle assessment did not significantly change (Fig. 4a), but compliance significantly improved $(\mathrm{P}<0.001$; Fig. $4 \mathrm{~b})$. Compliance and changes in $\mathrm{HbAlc}$ were not correlated with each other (data not shown). Switching from D to W did not result in serious adverse events, indicating satisfactory tolerability.

8

Baseline 8 weeks

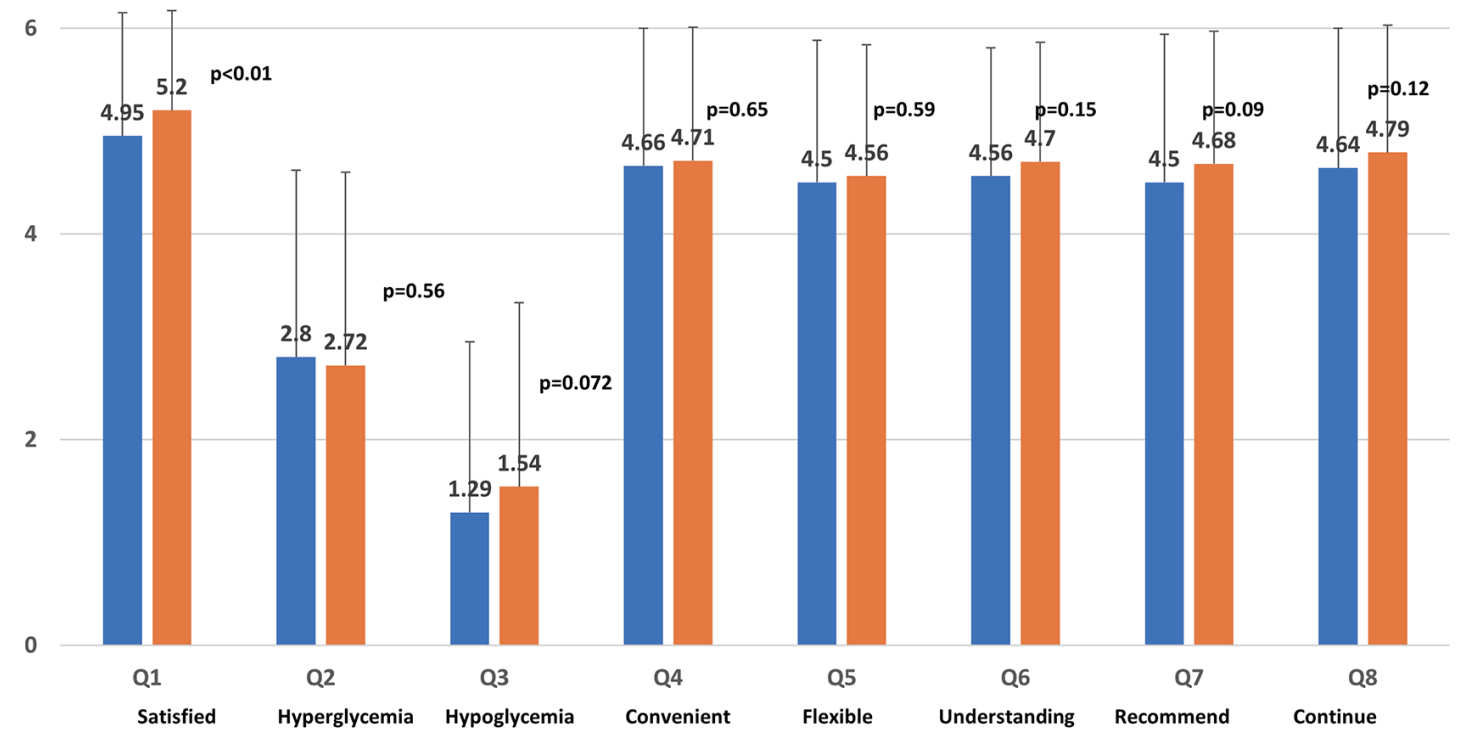

Figure 3. Score on the Diabetes Treatment Satisfaction Questionnaire. 
Table 4. Comparison of Clinical Parameters Between Patients With Increased and With Decreased Satisfaction Based on Q1 Questions

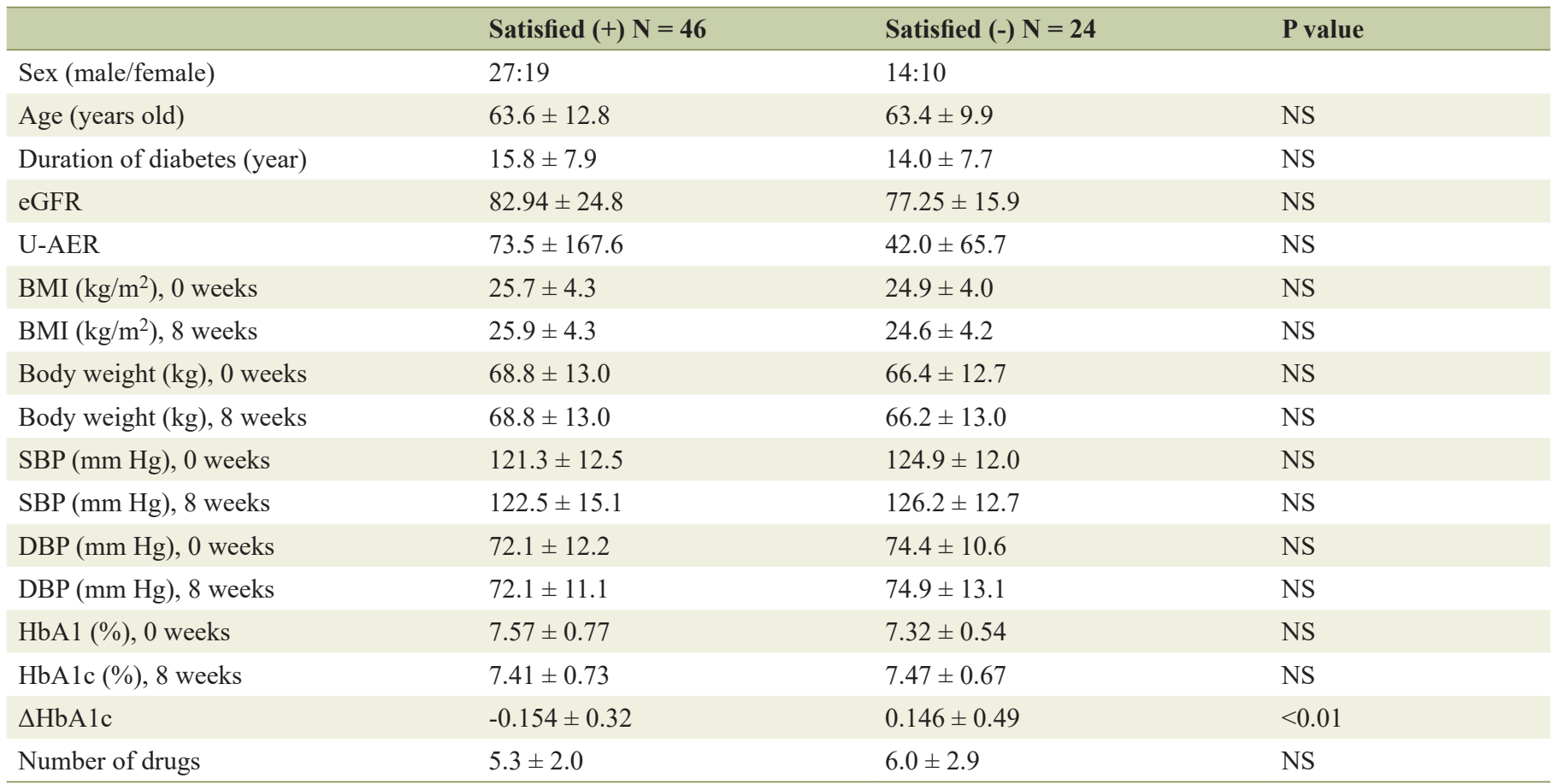

Data are presented as mean \pm SD. BMI: body mass index; SBP: systolic blood pressure; DBP: diastolic blood pressure; NS: not significant by Student's paired $t$-test.

\section{Discussion}

In this study, we proposed switching from $\mathrm{D}$ to $\mathrm{W}$; accordingly, $90.6 \%$ of patients requested to switch to $\mathrm{W}$ while $10.4 \%$
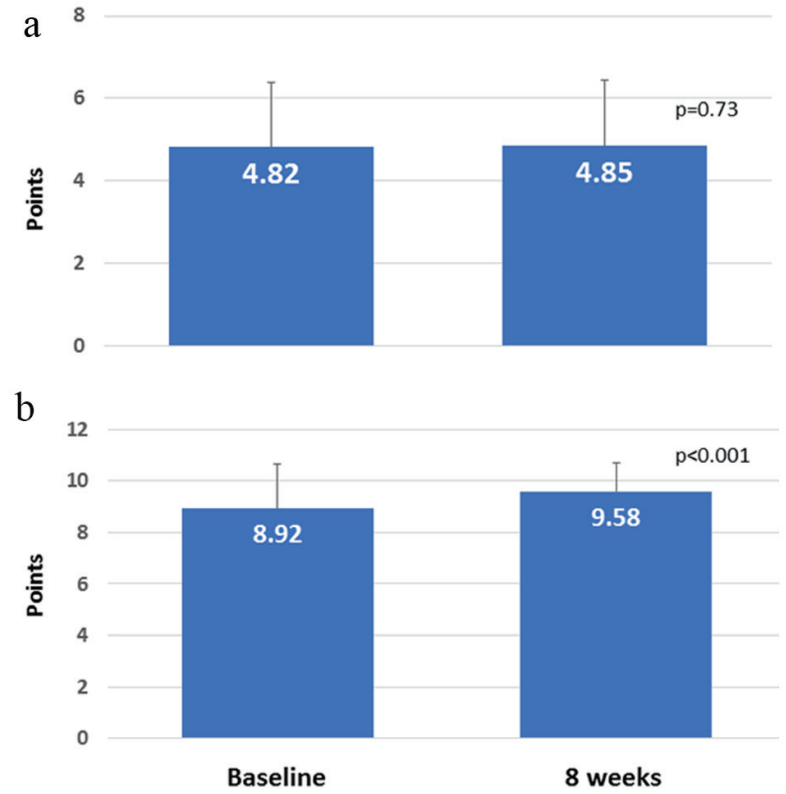

Figure 4. (a) Score for lifestyle assessment. (b) Compliance with medication schedule. did not. A previous survey in Japanese type 2 diabetic patients regularly visiting the outpatient clinic reported that $55.3 \%$ of patients requested a switch to $\mathrm{W}[7]$. The request rate may have been lower than that in our study because the survey was conducted during a 2-week limited prescription period (In Japan, only 2 weeks' worth of new drug can be prescribed the first year after its approval). Okazaki et al [8] recently reported that $81 \%$ of the 68 Japanese type 2 diabetic patients taking $\mathrm{D}$ requested to switch to $\mathrm{W}$, and that the elevation in $\mathrm{HbA} 1 \mathrm{c}$ levels before switching led patients to request the switch. In other words, patients with worse blood glucose levels requested switching to $\mathrm{W}$. In this study, changes in HbAlc levels were not evaluated before the medication switch. However, our study took place between June and August when there is seasonal variation (HbA1c levels are highest in winter-spring and lowest in summer-autumn in most patients in Japan) [9]. Considering this, it is unlikely that HbAlc was exacerbated in many patients in this study. Thus, although most patients requested switching to $\mathrm{W}$, it is unknown if the reason was due to increased $\mathrm{HbA} 1 \mathrm{c}$ levels.

In this study, significant changes in $\mathrm{HbAlc}$, body weight and BMI were not observed 8 weeks after switching from D to W. A meta-analysis of omarigliptin (five studies) and trelagliptin (two studies) [4] (follow-up of 12 weeks $(\mathrm{n}=2)$ or 24 weeks $(\mathrm{n}=5))$ also showed similar results. W was originally developed to have the same therapeutic effects as D. All previous reports involved clinical trials, which took place in a specialized environment where participants strictly followed the medication schedule; thus, the effects in patients were 
postulated to be equivalent. In actual clinical practice, on the other hand, it has been reported that drug adherence rates are less than $70 \%$ in diabetic patients [10]. Thus, a decrease in HbAlc level was anticipated through improved adherence and decreased incidence of forgetting to take the medication by switching to $\mathrm{W}$; however, the results were unchanged. There are two plausible reasons for this. First, the effects of W on adherence may not be substantial enough to improve blood glucose. Second, participants of this study already had good compliance, so switching to $\mathrm{W}$ may not have induced significant changes in $\mathrm{HbA} 1 \mathrm{C}$.

Patient adherence to $\mathrm{W}$ was evaluated with DTSQ. A significant difference was only observed for Q1 regarding satisfaction. Tosaki et al [11] investigated 26 type 2 diabetic patients and found significant differences in convenience (Q4) and flexibility (Q5) when patients switched from various types of D to W. In their study, D included twice-daily DPP-4 inhibitors. With the exception of the effect of switching from twicedaily to once-weekly medications, their study may not have reflected the true advantage of $\mathrm{W}$. In our study, all patients ( $\mathrm{n}$ $=153$ ) switched from D (once-daily) to $\mathrm{W}$ (once-weekly), reflecting the effects of $\mathrm{W}$ in a simple manner. This may have led to divergent results between different studies.

Patients with increased satisfaction after switching from D to $\mathrm{W}$ exhibited improved HbA1c levels. It is possible that patient satisfaction increased because the post-switch survey was conducted after informing them of the HbA1c levels measured that day, and patients with improved blood glucose levels may have been in a better mood. It is unclear if this was an effect of $\mathrm{W}$ itself.

Because type 2 diabetic patients often present with symptoms such as hypertension and dyslipidemia, the number of medications inevitably increase. We previously investigated drug adherence in patients who switched from taking two drugs to one combination tablet [12]. In the question, "Was your mental burden alleviated with one fewer medications?", approximately $80 \%$ of patients responded that the burden "greatly decreased" or "somewhat decreased." Before conducting this survey, healthcare providers did not anticipate any changes by reducing the number of tablets taken by patients by just one; however, the results indicated that patients were much more satisfied, even with this small decrease. Participants of this study took an average of 5.1 types of medications. Many patients who switched to W commented that, "this is so much easier!" By switching from D to W, patients took one fewer tablet for 6 days of the week, which may have alleviated their mental burden and contributed to their increased satisfaction.

W did not affect the score for lifestyle assessment. It has been reported that this score decreased in patients who showed attenuated therapeutic effects of $\mathrm{D}$ during the treatment course [13]. In other words, when there is a disturbance in a patient's lifestyle, the therapeutic effect of D declines. In this study, we determined whether $\mathrm{W}$ could improve lifestyle; however, it did not appear to have enough power to change this parameter.

Although medication adherence significantly improved with $\mathrm{W}, \mathrm{HbA} 1 \mathrm{c}$ levels did not improve. Moreover, medication adherence was not correlated with satisfaction. Because medication adherence was already high $(\geq 80 \%)$ when patients were taking D, even if adherence further improved by switching to $\mathrm{W}$, it is unlikely that the changes would be substantial enough to affect $\mathrm{HbA} 1 \mathrm{c}$ or satisfaction.

Both D and W are DPP-4 inhibitors with the same mechanisms of action, and as such, are viewed as the same type of drug by healthcare providers. However, they may be completely different drugs (once-daily drug versus once-weekly drug) from the patient's perspective. The results of this study showed that patient satisfaction and compliance improved with $\mathrm{W}$ compared to D. Therefore, it is important to present options to patients before prescribing medications.

\section{Conclusions}

This study revealed that $90 \%$ of patients taking D elected to switch to W. Moreover, patient satisfaction and compliance improved after switching to W. Increased satisfaction appeared to be influenced by improved blood glucose levels but was not associated with compliance. Switching from $\mathrm{D}$ to $\mathrm{W}$ did not affect $\mathrm{HbA} 1 \mathrm{c}$ levels but improved patient adherence.

\section{Acknowledgments}

The authors would like to acknowledge the patients and staff at participating sites for this study. The authors would also like to thank Forte Science Communications (https://www.fortescience.com) for their English language review of the manuscript.

\section{Conflicts of Interest}

The authors have no conflicts of interest to declare.

\section{Disclosure}

This study was accepted for oral presentation at the 61st Annual Meeting of the Japan Diabetes Society in Tokyo on May $24-26,2018$.

\section{References}

1. Oishi M, Yamazaki K, Okuguchi F, Sugimoto H, Kanatsuka A, Kashiwagi A, Japan Diabetes Clinical Data Management Study Group. Changes in oral antidiabetic prescriptions and improved glycemic control during the years 2002-2011 in Japan (JDDM32). J Diabetes Investig. 2014;5(5):581-587.

2. Inagaki N, Onouchi H, Maezawa H, Kuroda S, Kaku K. Once-weekly trelagliptin versus daily alogliptin in Japanese patients with type 2 diabetes: a randomised, doubleblind, phase 3, non-inferiority study. Lancet Diabetes Endocrinol. 2015;3(3):191-197.

3. Gantz I, Okamoto T, Ito Y, Okuyama K, O'Neill EA, Kaufman KD, Engel SS, et al. A randomized, placebo- 
and sitagliptin-controlled trial of the safety and efficacy of omarigliptin, a once-weekly dipeptidyl peptidase-4 inhibitor, in Japanese patients with type 2 diabetes. Diabetes Obes Metab. 2017;19(11):1602-1609.

4. Yamada T, Shojima N, Noma H, Yamauchi T, Kadowaki T. Weekly Versus Daily Dipeptidyl Peptidase 4 Inhibitor Therapy for Type 2 Diabetes: Systematic Review and Meta-analysis. Diabetes Care. 2018;41(4):e52-e55.

5. Ishii H, Bradley C, Riazi A, Barendse S, Yamamoto T. Japanese version of the Diabetes Treatment Satisfaction Questionnaire (DTSQ): translation and clinical evaluation. J Clin Exp Med. 2000;192:809-814.

6. Fujii H, Muto T, Haruyama Y, Nakade M, Kobayashi E, Ishisaki K, Yamasaki A. Community-based lifestyle modification of cardiovascular disease risks in middleaged Japanese: a 27-month update. Tohoku J Exp Med. 2010;220(4):307-318.

7. Suzuki D, Kuroki H, Tsukada A, Ito Y, Iyori N, Tosaka M, Tanaka S, et al. Does a patient choose once-weekly DPP4 inhibitor? Prog Med. 2016;36:389-392. (in Japanese).

8. Okazaki Y, Kadowaki T. [Examination of factors influencing the change from daily to weekly DPP-4 inhibitor.]
Diabetes Frontier. 2017;28(6):707-711. (in Japanese).

9. Sakura H, Tanaka Y, Iwamoto Y. Seasonal fluctuations of glycated hemoglobin levels in Japanese diabetic patients. Diabetes Res Clin Pract. 2010;88(1):65-70.

10. Manteuffel M, Williams S, Chen W, Verbrugge RR, Pittman DG, Steinkellner A. Influence of patient sex and gender on medication use, adherence, and prescribing alignment with guidelines. J Womens Health (Larchmt). 2014;23(2):112-119.

11. Tosaki T, Kamiya H, Yamamoto Y, Himeno T, Kato Y, Kondo M, Yamada Y, et al. Efficacy and patient satisfaction of the weekly DPP-4 inhibitors trelagliptin and omarigliptin in 80 Japanese patients with type 2 diabetes. Intern Med. 2017;56(19):2563-2569.

12. Suzuki K, Suzuki H, Morikawa H. [Effect of amlodipine besylate/atorvastatin calcium on type 2 diabetic patients with hypertension and dyslipidemia.] Prog Med. 2011;31:1781-1786. (in Japanese).

13. Tajiri Y, Tsuruta M, Ohki T, Kato T, Sasaki Y, Tanaka K, Kono S, et al. Long-term efficacy of sitagliptin for the treatment of type 2 diabetic patients in Japan. Endocr J. 2012;59(3):197-204. 